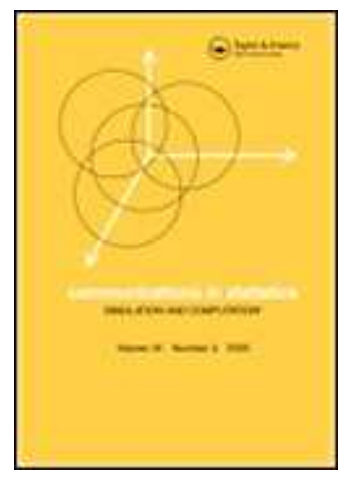

\title{
A Simple Bootstrap Method for Time Series
}

\begin{tabular}{|c|c|}
\hline Journal: & Communications in Statistics - Simulation and Computation \\
\hline Manuscript ID: & LSSP-2010-0267.R1 \\
\hline Manuscript Type: & Original Paper \\
\hline $\begin{array}{r}\text { Date Submitted by the } \\
\text { Author: }\end{array}$ & 14-Feb-2011 \\
\hline Complete List of Authors: & $\begin{array}{l}\text { Cai, Yuzhi; University of Plymouth, School of Computing and } \\
\text { Mathematics }\end{array}$ \\
\hline Keywords: & bootstrap, m-runs, threshold, run process \\
\hline Abstract: & $\begin{array}{l}\text { In this paper we present a simple bootstrap method for time series. } \\
\text { The proposed method is model free, and hence it enables us to } \\
\text { avoid } \\
\text { certain situations where the bootstrap samples may contain } \\
\text { impossible values due to resampling from the residuals. The } \\
\text { method } \\
\text { is easy to implement and can be applied to both stationary and } \\
\text { non-stationary time series. The simulation results and the } \\
\text { application to real time series data show that the method works } \\
\text { very } \\
\text { well. }\end{array}$ \\
\hline \multicolumn{2}{|c|}{$\begin{array}{l}\text { Note: The following files were submitted by the author for peer review, but cannot be converted } \\
\text { to PDF. You must view these files (e.g. movies) online. }\end{array}$} \\
\hline boottrap.zip & \\
\hline
\end{tabular}




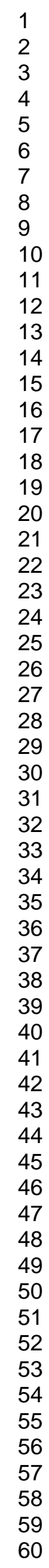

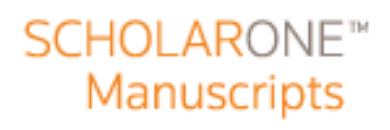

10

12

13

16

17

18

19

20

22

23

25

26

28

29

32

33

34

35

36

39

40

41

42

44

45

46

47

48

50

51

52 


\title{
A simple bootstrap method for time series
}

\author{
Yuzhi Cai* \\ University of Plymouth, $U K$
}

\begin{abstract}
In this paper we present a simple bootstrap method for time series. The proposed method is model free, and hence it enables us to avoid certain situations where the bootstrap samples may contain impossible values due to resampling from the residuals. The method is easy to implement and can be applied to both stationary and non-stationary time series. The simulation results and the application to real time series data show that the method works very well.
\end{abstract}

\section{Introduction}

The bootstrap and jackknife methods (Efron $(1979,1982))$ have proven to be powerful tools for approximating the sampling distribution and variance of complicated statistics defined on a sequence of independently identically distributed (i.i.d.) random variables. However, under dependence, the construction of sampling distribution is much more complicated and is far less obvious than that in independent cases. Several approaches have been proposed to extend the bootstrap to the situation where the assumption of i.i.d. random variables is violated. One approach is to reduce the problem to an approximate i.i.d. setting by focusing on the "residuals" of some general regression. Such examples include linear regression (Freedman (1981) and Liu (1988)), autoregressive time series (Efron and Tibshirani (1986) and Swanepoel and Van Wyk (1986)), nonparametric regression and nonparametric kernel spectral estimation (Härdle and Bowman (1988) and Franke and Härdle (1992)). In all these situations it is the residuals that are resampled, not the original observations. Another approach is to resample the original data in some way. For example, Künsch (1989) and independently, Liu and Singh (1988) presented a moving blocks bootstrap method, which constructs replicates by resampling partially overlapping blocks of observations. Later, Politis and Romano (1992) generalized the moving blocks resampling scheme and showed that their method is valid for general linear statistics that are asymptotically normal and consistent for a parameter of the whole joint distribution. A review paper on block, sieve and local bootstrap methods for time series and related theoretical aspects of the procedures

*Address for correspondence: Dr Yuzhi Cai, School of Computing and Mathematics, University of Plymouth, Plymouth PL4 8AA, United Kingdom. Email: ycai@plymouth.ac.uk 
as well as their performance on finite-sample data was given by Bühlmann (2002). It is interesting to see that Kim et al. (1993) introduced a binary bootstrap method for inference with autocorrelated binary data. This method differs from the moving block approach in that it lets the data divide itself into blocks of random length, consisting of runs of 0's and 1's. Later they extended the method to general time series by way of the threshold bootstrap method, see, for example, Park and Willemain (1999), Park et al (2001).

In this paper, we propose a model free bootstrap (MFB) method which can be used to any time series. In Section 2 we present the method and in Section 3 we provide simulation results. An interesting application to real time series is given in Section 4. Finally, some comments and conclusions are given in Section 5.

\section{The method}

For binary time series we have two runs: 0-runs containing consecutive 0's, and 1-runs containing consecutive 1's. For general time series, we define $m$-runs according to the chosen thresholds, and each run also contains consecutive observations of random length.

Suppose an observed time series is given by $x_{1}, x_{2}, \ldots, x_{n}$. Let $T_{i}(i=1, \ldots, m)$ be the thresholds such that $-\infty<T_{1}<T_{2}<\cdots<T_{m-1}<T_{m}=\infty$. The values of the thresholds can be decided as follows. Let $h=1 / m$, then $T_{i}=(i h)$ th quantile of $x_{1}, x_{2}, \ldots, x_{n}$ for $i=1, \ldots, m-1$, and $T_{m}=\infty$.

Let $y_{t}=i$ if $i$ is the smallest value such that $x_{t} \leq T_{i}$. Then the MFB method consists of the following several steps.

1. Turn the time series $x_{t}$ into a process $y_{t}$.

For example, suppose $x_{t}$ is given by

$$
x_{t}: \quad 2,7,0,0,3,1,6,5,9,8,0,3,5,4,7,9,1,0,1 .
$$

Let $m=3$, then we have $T_{1}=1, T_{2}=5, T_{3}=\infty$. Hence the corresponding $y_{t}$ process is given by

$$
y_{t}: \quad 2,3,1,1,2,1,3,2,3,3,1,2,2,2,3,3,1,1,1 .
$$

2. Break the series $x_{t}$ and $y_{t}$ into a sequence of 1 -runs, ..., $m$-runs.

$$
\begin{array}{ll}
\tilde{x}_{t}: & (2)(7)(0,0)(3)(1)(6)(5)(9,8)(0)(3,5,4)(7,9)(1,0,1) \\
\tilde{y}_{t}: & (2)(3)(1,1)(2)(1)(3)(2)(3,3)(1)(2,2,2)(3,3)(1,1,1) .
\end{array}
$$

3. Create a bootstrap sample of $\tilde{y}_{t}$ by sampling with replacement from the pools of 1runs, 2-runs ,..., $m$-runs according to the order of runs given, for example, in (1). Repeat this sampling procedure until the size of the bootstrap sample is at least the same as that of $y_{t}$. 
In the above example, we should sample with replacement from the pools of 2-runs, 3-runs, 1-runs and so on, and repeat this procedure until the sample size is at least 19. One bootstrap sample from the run process $\tilde{y}_{t}$ could be

$$
y_{t}^{*}:(2)(3,3)(1)(2)(1)(3,3)(2)(3,3)(1,1)(2)(3)(1)(2)(3)(1,1,1) .
$$

Note that the last three runs (2) and (3) and $(1,1,1)$ are the samples obtained from the pool of 2-runs, 3-runs and 1-runs respectively, which correspond to the first three runs of $\tilde{y}_{t}$. These three extra runs are required because after we have sampled all the runs given in $\tilde{y}_{t}$ the size of the bootstrap sample of $y_{t}$ process is still less than 19 . It is also worth mentioning that those extra runs do not have significant effects on the bootstrap samples as usually only several values at the end of a sample are required.

4. Convert the runs into the values of the original time series and truncate from the final run to insure that the bootstrap sample size is the same as that of the original sample size.

Note that the mapping between $\tilde{y}_{t}$ and $\tilde{x}_{t}$ is one-to-one. Hence in the above example, the corresponding bootstrap sample from the run process $\tilde{x}_{t}$ is

$$
x_{t}^{*}:(3)(9,8)(0)(2)(1)(7,9)(5)(9,8)(0,0)(3)(6)(0)(5)(7)(1,0,1)
$$

By truncating from the last run a bootstrap sample of $x_{t}$ is given by

$$
3,9,8,0,2,1,7,9,5,9,8,0,0,3,6,0,5,7,1
$$

5. Goto step 3 until $M$ bootstrap samples are obtained.

It is worth mentioning that the MFB method guarantees that the local autocorrelation structures of a bootstrap sample are similar to those of the observed time series because the unit of the bootstrapping is runs. Furthermore, the MFB method also guarantees that the overall evolution of a bootstrap sample is similar to that of the original time series because the order of the runs in the observed time series is the same as that in the bootstrap samples.

On the other hand, the way that the threshold values are determined guarantees that a run within a certain quantile range of $x_{t}(t=1, \ldots, n)$ will be replaced by a random sample from a pool of runs within the same quantile range. Hence a bootstrap sample will not only maintain a similar autocorrelation structure but also have similar proportion of samples in each quantile range of $x_{t}$ defined by the thresholds.

Therefore, we would expect that the MFB method should provide us a means of estimating the distribution of a complicated statistic of interest. In the rest of the paper we will carry out extensive simulation studies in order to check the performance of the method. We will also compare our MFB method with the moving block bootstrap method. It is worth mentioning that although we found it difficult to show some theoretical results on the bootstrap estimates obtained from the proposed MFB method, we will carry out further investigations on this issue in the future. 


\section{Simulation studies}

Simulation study 1. MFB for i.i.d. data.

It is well known that the variance of $\bar{X}=\frac{1}{n} \sum_{i=1}^{n} X_{i}$ is given by $\sigma_{\bar{X}}^{2}=\sigma^{2} / n$, where $X_{1}, \ldots, X_{n}$ are $n$ i.i.d. random variables with variance $\sigma^{2}$. Given a random sample $x_{1}, \ldots, x_{n}, \sigma_{\bar{X}}^{2}$ may be estimated by $\hat{\sigma}_{\bar{x}}^{2}=\hat{\sigma}^{2} / n$, where $\hat{\sigma}^{2}$ is the sample variance. In this simulation study, we use MFB method to study the bootstrap distribution of $\hat{\sigma}_{\bar{x}}^{2}$ and to compare its mean with the true value of $\sigma_{\bar{X}}^{2}$.

Specifically, 50 independent random samples $x_{1}^{(j)}, \ldots, x_{200}^{(j)}(j=1, \ldots, 50)$ were simulated from the normal distribution $N\left(0,4^{2}\right)$. Then we have $n=200$ and $\sigma_{\bar{X}}^{2}=16 / 200=$ 0.08 .

For each $j$, a sequence of $m$ numbers of thresholds were considered: Let $m=m_{\ell}$ $(\ell=1,2, \ldots, L)$ be the sequence of numbers of thresholds considered in this simulation study, and let $m_{\ell}$ be one of the following numbers: $2,4,6,8,10,12,14,16,18,20,30,40$, $50,60,70,80$ and 90 . Hence we have $\ell=1,2, \ldots, 17$.

For each $j$ and $\ell, 500$ bootstrap samples of size 200 were obtained by using the MFB method, and then $\sigma_{\bar{X}}^{2}$ were estimated by $\hat{\sigma}_{\bar{x}_{(j \ell k)}}^{2}=\hat{\sigma}_{(j \ell k)}^{2} / n$, where $\hat{\sigma}_{(j \ell k)}^{2}$ is the sample variance of the $k$ th bootstrap sample, $k=1, \ldots, 500$. Then $\hat{\sigma}_{\bar{x}_{(j \ell k)}}^{2}(k=1, \ldots, 500)$ represents an empirical bootstrap distribution of $\hat{\sigma}_{\bar{x}}^{2}$.

For each $j$ and $\ell$, let

$$
\hat{\sigma}_{\bar{x}_{(j \ell \cdot)}}^{2}=\frac{1}{500} \sum_{k=1}^{500} \frac{\hat{\sigma}_{(j \ell k)}^{2}}{n}, j=1, \ldots, 50, \ell=1, \ldots, 15,
$$

and let $q_{j \ell}^{0.025}$ and $q_{j \ell}^{0.975}$ be the $2.5 \%$ and $97.5 \%$ quantiles of $\hat{\sigma}_{\bar{x}_{(j \ell k)}}^{2}(k=1, \ldots, 500)$ respectively. Then $\hat{\sigma}_{\bar{x}(j \ell)}^{2}$ gives the mean of the bootstrap estimate of $\sigma_{\bar{X}}^{2}$ for the $j$ th data set with $m_{\ell}$ numbers of thresholds, and $\left(q_{j \ell}^{0.025}, q_{j \ell}^{0.975}\right)$ forms a corresponding $95 \%$ bootstrap quantile range.

Now by letting

$$
\hat{\sigma}_{\bar{x}_{(\ell \ell)}}^{2}=\frac{1}{50} \sum_{j=1}^{50} \hat{\sigma}_{\bar{x}_{(j \ell \cdot)}}^{2}, \quad \hat{q}_{\ell}^{0.025}=\frac{1}{50} \sum_{j=1}^{50} q_{j \ell}^{0.025}, \quad \hat{q}_{\ell}^{0.975}=\frac{1}{50} \sum_{j=1}^{50} q_{j \ell}^{0.975},
$$

we can see the average performance of the bootstrap estimates of $\sigma_{\bar{X}}^{2}$ as a function of the number of thresholds required by the developed MFB method. Figure 1 shows the plot of $\hat{\sigma}_{\bar{x}(\cdot .)}^{2}$ against the number of thresholds, together with the corresponding $95 \%$ bootstrap quantile ranges. It is seen that the width of the $95 \%$ bootstrap quantile range tends to be more stable as $\ell$ increases. However, the average bootstrap estimate of $\sigma_{\bar{X}}^{2}$ seems to be independent of the number of thresholds, which is what we should have expected because we are dealing with independent data in this simulation study. The mean squared error (MSE) between $\hat{\sigma}_{\cdot \ell}^{2}$ and the true value $\sigma_{\bar{X}}^{2}=0.08$ over the range of the numbers of thresholds is $6.75 \times 10^{-6}$ which is very small. Figure 1 also suggest that on average, 40 threshold values 


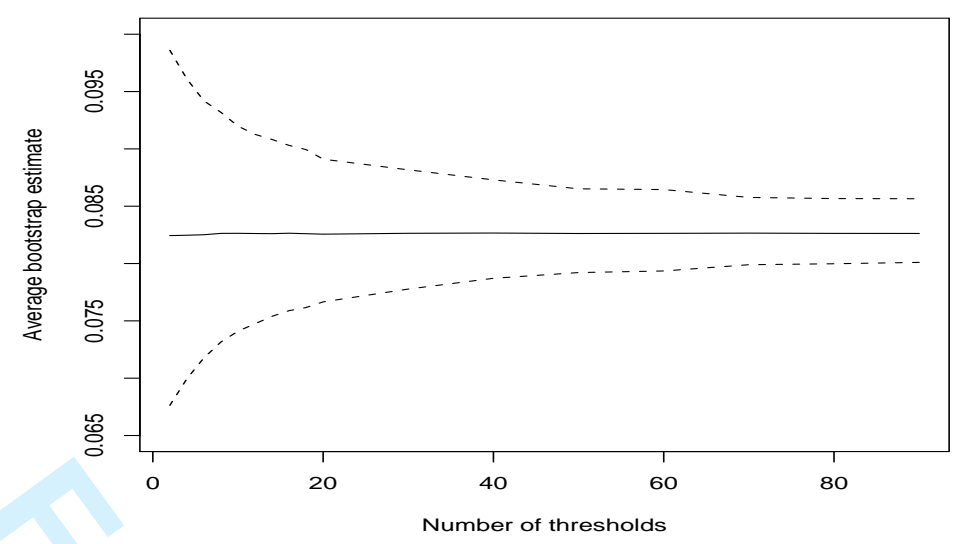

Figure 1: Plot of the average bootstrap estimates of $\sigma_{\bar{X}}^{2}$ (continuous curve) over 50 independently simulated data sets against the number of thresholds in Simulation study 1 . The two dashed curves correspond to the average $95 \%$ bootstrap quantile range for $\sigma_{\bar{X}}^{2}$.

would be enough for this simulation study as both the estimates of $\sigma_{\bar{X}}^{2}$ and the corresponding $95 \%$ bootstrap quantile ranges tend to be stable for any $m_{\ell} \geq 40$.

Simulation study 2. MFB for time series.

Consider an $\operatorname{ARMA}(2,1)$ time series model

$$
x_{t}=0.8 x_{t-1}-0.6 x_{t-2}+\varepsilon_{t}+0.3 \varepsilon_{t-1},
$$

where $\varepsilon_{t}$ are i.i.d. normal random variables with mean 0 and variance 2 . Hence we have $\phi_{1}=0.8, \phi_{2}=-0.6$ and $\phi_{3}=0.3$. In this simulation study, we investigate the average performance of the bootstrap estimates and the corresponding bootstrap quantile ranges for the model parameters.

Similar to the Simulation study 1,50 independent time series $x_{1}^{(j)}, \ldots, x_{200}^{(j)}(j=1, \ldots$, 50) were simulated from (2).

For each $j$, a sequence of numbers of thresholds were considered. In this simulation study, we also let $m_{\ell}$ take the following values: $2,4,6,8,10,12,14,16,18,20,30,40,50$, 60, 70, 80 and 90.

For each $j$ and $\ell, 500$ bootstrap samples of size 200 were obtained by using the MFB method and then an $\operatorname{ARMA}(2,1)$ model was fitted to each of the bootstrap samples by using the maximum likelihood estimation method. Let $\phi_{j \ell k}^{(i)}$ be the maximum likelihood estimate (MLE) of $\phi_{i}$, where $i=1,2,3, j=1, \ldots, 50, \ell=1, \ldots, 17$ and $k=1, \ldots, 500$. Then an average bootstrap estimate of $\phi_{i}$ may be given by

$$
\phi_{j \ell .}^{(i)}=\frac{1}{500} \sum_{k=1}^{500} \phi_{j \ell k}^{(i)}, \quad i=1,2,3, j=1, \ldots, 50, \ell=1, \ldots, 17 .
$$


Let $q_{j \ell}^{(i) 0.025}$ and $q_{j \ell}^{(i) 0.975}$ be the $2.5 \%$ and $97.5 \%$ quantiles of $\phi_{j \ell k}^{(i)}(k=1, \ldots, 500)$ respectively. Then $\left(q_{j \ell}^{(i) 0.025}, q_{j \ell}^{(i) 0.975}\right)$ forms a $95 \%$ bootstrap quantile range of $\phi_{i}$ for each $j$ and $\ell$. Furthermore, let

$$
\phi_{\cdot \ell \cdot}^{(i)}=\frac{1}{50} \sum_{j=1}^{50} \phi_{j \ell \cdot,}^{(i)}, \quad q_{\ell}^{(i) 0.025}=\frac{1}{50} \sum_{j=1}^{50} q_{j \ell}^{(i) 0.025}, \quad q_{\ell}^{(i) 0.975}=\frac{1}{50} \sum_{j=1}^{50} q_{j \ell}^{(i) 0.975} .
$$

Then $\phi_{\cdot \ell \cdot}^{(i)}$ and $\left(q_{\ell}^{(i) 0.025}, q_{\ell}^{(i) 0.975}\right)$ provide an average bootstrap estimate of $\phi_{i}$ and an average 95\% bootstrap quantile range over 50 independent time series from model (2) for each $\ell$.

The left column of Figure 2 shows the plots of $\phi_{\cdot \ell}^{(i)}$ against the number of thresholds (continuous curves) together with the $95 \%$ bootstrap quantile ranges (dashed curves), which are compared with the true parameter values (darker horizontal lines) and the average $95 \%$ confidence intervals (lighter horizontal lines) of $\phi_{i}$ obtained by using the MLE method over 50 independent time series from model (2).
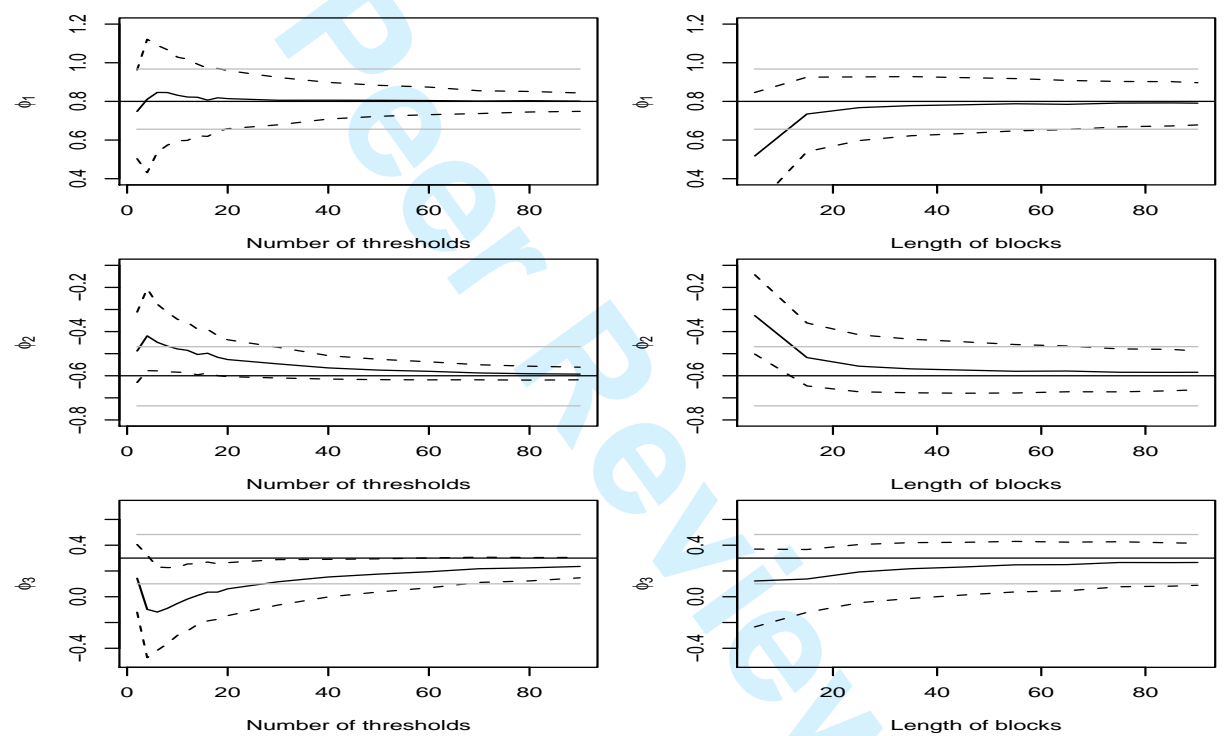

Figure 2: Plots of the true parameter values (darker horizontal lines), the average bootstrap estimates (continuous curves), 95\% bootstrap quantile ranges (dashed curves) and $95 \%$ confidence intervals (lighter horizontal lines) obtained by using the MLE method over 50 independently simulated time series from model (2) in Simulation study 2.

We also used the moving block bootstrap method to obtain 500 bootstrap samples for each $j$ and $J$, where $J$ is the length of the blocks. In this simulation study we let $J \in$ $\{5,15,25,35,45,55,65,75,85,90\}$. Similarly, we show the results in the right column of Figure 2 .

It is seen from the left column of Figure 2 that $\phi_{. \ell}^{(i)}$ converges to the true value of $\phi_{i}$ and the bootstrap quantile range also tends to contain the true values of $\phi_{i}$ for $i=1,2,3$ 
as the number of thresholds increases. Furthermore, the $95 \%$ bootstrap quantile ranges are gradually covered completely by the $95 \%$ confidence intervals obtained by using the MLE method. This may suggest that on average the bootstrap quantile range provides an improved measurement on the variation of the estimated model parameters as long as the number of thresholds is large enough.

It is also seen from the right column of Figure 2 that on average the performance of the moving block bootstrap method is quite similar to our MFB method except that the $95 \%$ bootstrap quantile ranges are wider than those obtained from the MFB method.

Our experience shows that the required number of thresholds is model dependent. While the optimal choice of the number of thresholds is certainly worth further investigations in the future, the simulation studies also suggest a practical means of deciding a proper number of thresholds for obtaining good bootstrap estimates. That is, we could take the number of thresholds as $m_{0}$ such that for any $m \geq m_{0}$ the bootstrap estimates of a statistic of interest and the corresponding bootstrap quantile ranges do not change significantly, which can be identified through plots like those shown in Figure 2. Therefore the developed MFB method enables us to obtain a required quantile range for the statistic of interest especially when it is difficult or impossible to obtain by using other methods including the MLE method. Our experience also shows that if the number of bootstrap samples are very large then the MFB method can be time consuming even for the values of the number of thresholds considered in this paper.

\section{Applications}

In this section, we applied the developed methodology to the Dow Jones Industrial Average (DJI) from 2 January 2004 to 8 October 2010. The length of the series is 1705 . The time series plots of the observed series and its log returns (in percentage), denoted by $y_{t}$ $(t=1, \ldots, n=1704)$ are given in Figure 3(a)(b). As expected, the observed DJI series shows occurrence of extremes and volatility clustering, which is further confirmed by the acf plot of $y_{t}$ and the acf plot of $y_{t}^{2}$ in Figure 3(c)(d). Therefore, we fitted a GARCH $(1,1)$ model to $y_{t}$ by using the MLE method. Let $r_{t}$ be the residual series of the fitted model.

Figure 3(e)(f) show the acf plots of $r_{t}$ and $r_{t}^{2}$ respectively, from which we see that the fitted GARCH $(1,1)$ model has indeed explained much of the variations of the observed series. Other diagnostic tests also show that the fitted $\operatorname{GARCH}(1,1)$ model is a good candidate for this time series.

As our objective of this application is not to find the best time series model for this data set, we will use the fitted $\operatorname{GARCH}(1,1)$ model to investigate the performance of the developed MFB method. The fitted GARCH $(1,1)$ model is given by

$$
y_{t}=\sigma_{t} \varepsilon_{t}, \quad \sigma_{t}^{2}=\alpha_{0}+\alpha_{1} y_{t-1}^{2}+\alpha_{2} \sigma_{t-1}^{2},
$$

where $\varepsilon_{t}$ are i.i.d. $N(0,1)$, and the parameter estimates and the corresponding standard errors are given in Table 1 . 
Figure 3: Plots of (a) the DJI time series, (b) its log returns, (c) acf of $y_{t}$, (d) acf of $y_{t}^{2}$, (e) acf of $r_{t}$ and (f) acf of $r_{t}^{2}$.

\begin{tabular}{c|ccc}
\hline & $\alpha_{0}$ & $\alpha_{1}$ & $\alpha_{2}$ \\
\hline Estimates & 0.0126 & 0.0795 & 0.9089 \\
Standard errors & 0.0023 & 0.0099 & 0.0107 \\
\hline
\end{tabular}

Table 1: The estimated parameter values and the corresponding standard errors of the GARCH $(1,1)$ model for DJI time series.

Again, we consider a sequence of numbers of thresholds $m_{\ell}=2,4,6,8,10,12,14,16$, $18,20,30,40,50,60,70,80,90,100$ and 150, hence $\ell=1, \ldots, 19$. For each $\ell$, we obtain 500 bootstrap samples from the observed log return series $y_{t}$. The estimated parameter values based on the bootstrap samples are denoted by $\alpha_{i \ell k}(i=0,1,2, \ell=1, \ldots, 19$ and $k=1, \ldots, 500)$. Let $\alpha_{i \ell}=\frac{1}{500} \sum_{k=1}^{500} \alpha_{i \ell k}$, and $\left(q_{i \ell}^{0.025}, q_{i \ell}^{0.975}\right)$ be the average bootstrap estimate and a 95\% bootstrap quantile range of $\alpha_{i}$ respectively, where $q_{i \ell}^{0.025}$ and $q_{i \ell}^{0.975}$ are the 0.25 th and 97.5 th quantiles of $\alpha_{i \ell k}(k=1, \ldots, 500)$ respectively.

The left column of Figure 4 shows the plots of the bootstrap parameter estimates (continuous curves) and the corresponding 95\% bootstrap quantile ranges (dashed curves) for $\alpha_{i}(i=0,1,2)$ obtained by using the MFB method. It also shows the MLE of the model parameters (darker horizontal lines) and the corresponding 95\% confidence intervals (lighter horizontal lines). To make the figure clearer, we plotted the $\log \left(\alpha_{i}\right)(i=0,1)$ and $\alpha_{2}$ against the number of thresholds respectively. It is seen that initially the bootstrap estimates are not stable, but when the number of thresholds increases both the bootstrap estimates and the bootstrap quantile ranges converged such that the bootstrap estimates approach to the MLEs of the model parameters and the bootstrap quantile ranges tend to be within the confidence intervals obtained from the MLE method. It is worth mentioning that if we let 
$m_{0}=80$. Then for any $m_{\ell} \geq m_{0}$ all the bootstrap estimates are very similar, so are the corresponding $95 \%$ bootstrap quantile ranges. Therefore, these estimates may be used for further statistical analysis if required.

The right column of Figure 4 shows the similar results obtained by using the moving block bootstrap method. Again, the overall performance of the moving block bootstrap method is similar to that of the MFB method. That is, the bootstrap estimates of the model parameters converge to the MLEs of the model parameters as the length of blocks increases, but the corresponding $95 \%$ quantile ranges are wider than those obtained from MFB method.
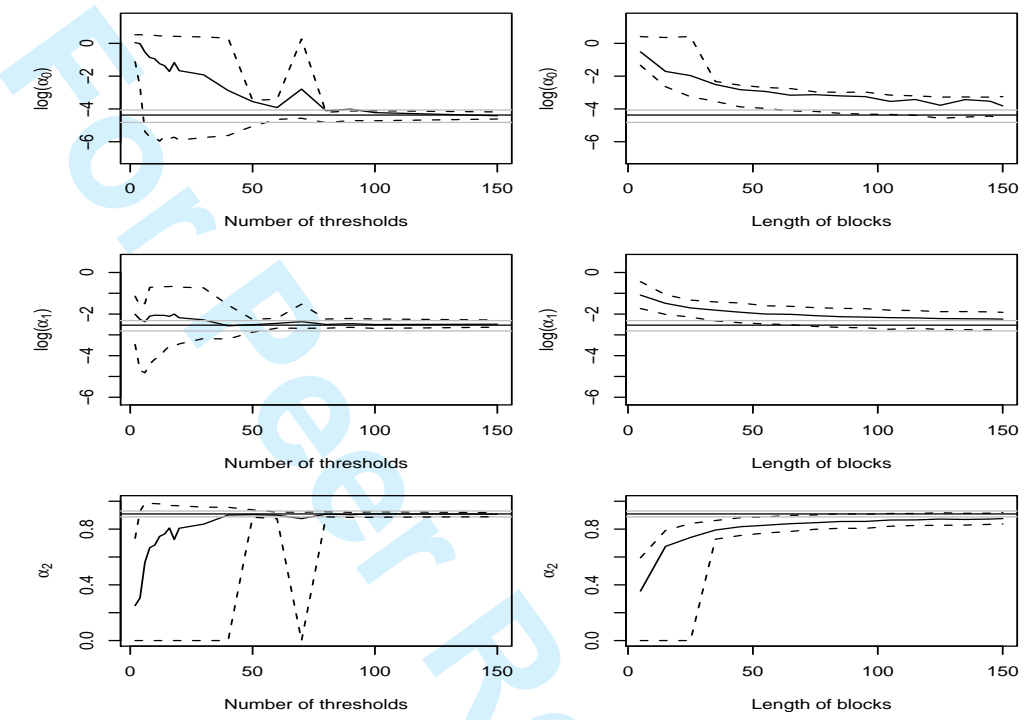

Figure 4: Plots of the bootstrap estimates and bootstrap quantile ranges together with the MLE of the model parameters and confidence intervals for DJI time series.

\section{Comments and conclusions}

We have presented a model free bootstrap method for time series, which is very easy to implement. Such a model-free bootstrap method enables us to avoid certain situations where the bootstrap samples may contain impossible values due to resampling from the residuals. Large amount of simulation studies have been carried out in order to investigate the performance of the developed MFB method. The simulation results show that as the number of thresholds increases, the bootstrap estimates of model parameters will converge to the true parameter values. Furthermore, the $95 \%$ bootstrap quantile ranges of the model parameters are on average narrower than the $95 \%$ confidence intervals obtained by using the MLE method if available. The empirical application to the DJI time series shows that the method also works well in practice. 


\section{Acknowledgement}

We sincerely thank the referees for their very constructive comments and suggestions which have significantly enhanced the quality and presentation of this paper.

\section{References}

[1] Bühlmann, P. (2002). Bootstraps for time series. Statistical Science, 17, 52-72.

[2] Efron, B. (1979). Bootstrap method : another look at the jackknife. The Annals of Statistics, 7, 1-26.

[3] Efron, B. (1982). The jackknife, the bootstrap, and other resampling plans. SIAM, CBMS-NSF Regional Conference Series in Applied Mathematics, 38, Philadelphia.

[4] Efron, B. and Tibshirani, R.J. (1986). Bootstrap methods for standard errors, confidence intervals and other measures of statistical accuracy. Statistical Science, 1, 5477.

[5] Freedman, D.A. (1981). Bootstrapping regression models. The Annals of Statistics, 9, 1218-1228.

[6] Frank , J. and Härdle W. (1992). On bootstrapping kernel spectral estimates. The Annals of Statistics, 20, 121-145.

[7] Härdle, W. and Bowman A. (1988). Bootstrapping in nonparametric regression : local adaptive smoothing and confidence bands. Journal of the American Statistical Association, 83, 100-110.

[8] Kim , Y.B., Haddock, J. and Willemain T.R. (1993). The binary bootstrap : inference with auto correlated binary data. Communications in Statistics - Simulation and Computation, 22(1), 205-216. 
[9] Künsch, H.R. (1989). The jackknife and the bootstrap for general stationary observations. The Annals of Statistics, 17, 1217-1241.

[10] Liu, R.Y. (1988). Bootstrap procedures under some non-i.i.d. models. The Annals of Statistics, 16, 1696-1708.

[11] Liu , R.Y. and Singh K. (1988). Moving blocks jackknife and bootstrap capture weak dependence. Unpublished Manuscript, Department of Statistics, Rutgers University.

[12] Park, D. and Willemain, T.R. (1999). The threshold bootstrap and threshold jackknife. Computational Statistics and Data Analysis, 31, 187-202.

[13] Park, D., Kim, Y.B., Shin, K. and Willemain, T.R. (2001). Simulation output analysis using the threshold bootstrap. European Journal of Operational Research, 134, 17-28.

[14] Politis, D.N. and Romano J.P. (1992). A general resampling scheme for triangular arrays of $\alpha$-mixing random variables with application to the problem of spectral density estimation. The Annals of Statistics, 20, 1985-2007.

[15] Swanepoel, J.W.H. and Van Wyk, J.W.J. (1986). The bootstrap applied to spectral density function estimation. Biometrika, 73, 135-142 . 